\title{
Transmit Power Control Mechanisms in IEEE 802.11 Cellular Networks
}

\author{
Elena Lopez-Aguilera, Jordi Casademont \\ Wireless Networks Group - Telematics Department \\ Barcelona, Spain \\ \{elopez, jordi.casademont\} @ entel.upc.es
}

\begin{abstract}
Actually, Wireless Local Area Networks (WLAN) are being extensively deployed in many places to provide easy access to Internet. Moreover, networks that span large buildings or campuses must comprise multiple cells. Surprisingly, multicell environments that correspond to realistic situations in highly deployed areas have received little attention in the literature.

If we compare the system performance in a multicell scenario with its behavior in an isolated single cell, we can observe an important performance decrease that becomes higher with the growth of the transmission data rate employed and due to cochannel interference.

In this paper, we propose some power control mechanisms to operate in IEEE 802.11 cellular networks. With the employment of such methods we aim at reducing the interference influence on network performance and at homogenizing the behavior of the stations placed at different spatial positions with respect to their access point. Results exposed show that the employment of such power control mechanisms leads to an important reduction in the interference level.
\end{abstract}

\section{Categories and Subject Descriptors}

C.2.1 [Computer Communication Networks]: Network Architecture and Design - wireless communication

\section{General Terms}

Algorithms, Performance

\section{Keywords}

Cellular networks, IEEE 802.11, WLAN, power control

\section{INTRODUCTION}

Since the advent of the first IEEE 802.11 standard, much research effort has been spent on the evaluation of its system performance. Actually, the idea of designing an outdoor cellular network based on WLAN IEEE 802.11 results very attractive, because IEEE 802.11 presents various advantages in front of $2.5 \mathrm{G}$ and $3 \mathrm{G}$

Permission to make digital or hard copies of all or part of this work for personal or classroom use is granted without fee provided that copies are not made or distributed for profit or commercial advantage and that copies bear this notice and the full citation on the first page. To copy otherwise, or republish, to post on servers or to redistribute to lists, requires prior specific permission and/or a fee.

IWCMC'06, July 3-6, 2006, Vancouver, British Columbia, Canada. Copyright 2006 ACM 1-59593-306-9/06/0007...\$5.00. wireless networks: the low cost of the equipment required, its operation in unlicensed spectrum and the higher data rates offered, which exceed the maximum rates provided by Enhanced Data Rates for GSM Evolution (EDGE) and Wideband Code Division Multiple Access (WCDMA) networks.

In [3] and [7] we find several analysis of propagation issues in outdoor environments. But a common aspect of these studies is that they are limited to a single cell scenario. In [8] we have evaluated the IEEE 802.11 network performance in a cellular environment, because multicell scenarios have received little attention in the literature. However, networks that span large buildings or campuses must comprise multiple cells. In the public $2.4 \mathrm{GHz}$ band there is a small number of available channels. Thus, co-channel and adjacent channel interferences suppose important issues. We have presented its performance under different load conditions and carried out a comparison between these results and the ones obtained in a single cell environment. We have observed that for high data rates the system throughput performance decreases considerably due to co-channel interference. Finally, each station performance in an infrastructure Basic Service Set (BSS) depends strongly of its relative position to its Access Point (AP). The throughput performance for stations becomes poorer with the distance increase.

In this way, in this paper we propose several power control mechanisms to reduce the interference influence on cellular network performance, to homogenize the behavior of the different stations and to assimilate their performance to the observed in a single cell environment.

Several power control schemes for IEEE 802.11 networks have been proposed in the literature. Some power control protocols have been presented in mobile ad-hoc networks with the objectives of improving energy consumption [6] and creating the connectivity set that increases system performance [13]. On the other hand, some studies have been carried out in infrastructure networks. In [11] a transmit power control mechanism is exposed, which saves energy in wireless devices. Finally, power and rate adaptation methods have been recently presented in ad-hoc [12] and in infrastructure networks [5].

In this paper we analyze the suitability of different power control mechanisms to work in a cellular IEEE 802.11 network. To the best of our knowledge, this study is the first proposal of transmit power control mechanisms to operate in such kind of scenarios.

The organization of the rest of the paper is as follows: section II describes the simulation environment, section III presents the power control proposals and section IV exposes the main results of IEEE 802.11 cellular network performance employing these 
mechanisms. Finally section V concludes with the most relevant points of the paper.

\section{SIMULATION ENVIRONMENT DESCRIPTION}

We have chosen the physical layer of the IEEE 802.11g to perform our study. We employ a simulation tool implemented in UPC (Technical University of Catalonia), which is written in $\mathrm{C}++$ programming language and follows all the IEEE 802.11 protocol details. It emulates as closely as possible the real operation of each transmitting station. Our simulation tool permits the IEEE 802.11 protocol emulation in a single cell environment and in a cellular network. On the contrary, we have observed that the wellknown NS-2 Simulator presents some flaws in a cellular scenario. In this way, we choose the exposed simulation tool to study cellular network performance. It has been validated comparing the results obtained with the information published in [1], under identical simulation conditions.

We restrict our investigation to infrastructure networks operating in Distributed Coordination Function (DCF) mode. The simulation environment consists of 100 hexagonal cells, which form a rectangular area, and only the 36 middle cells are taken to compute the statistics, in order to mitigate the border effect. Each BSS has a cell radius of $200 \mathrm{~m}$ and is composed of $1 \mathrm{AP}$ and 10 user stations, which are distributed randomly following a uniform distribution throughout the cell area. All cells have the stations distributed at the same positions, which are static. Only user stations transmit data frames with a constant payload size of 1023 bytes, and the time between consecutive arrivals follows an exponential distribution function. The simulation time employed is large enough, so that we achieve the convergence of the power control algorithms. Besides the interference, each receiver is affected by a noise power of $-96 \mathrm{dBm}$ [7].

As path loss model we employ the validated propagation model for IEEE 802.11 devices operating at $2.4 \mathrm{GHz}$ in outdoor environments specified in [3].

We consider independent errors occurring during transmission with an upper bound on the frame error probability under the assumption of binary convolutional coding and hard-decision Viterbi decoding [10].

\section{TRANSMIT POWER CONTROL PROPOSALS}

In [8] we have observed that throughput performance in a cellular network decreases considerably for high transmission data rates, because of the influence of co-channel interfering frames. Stations at different spatial positions with respect to an access point may experience different transmission conditions: a station far away from the access point will have higher error rates, and consequently lower Signal to Noise and Interference Ratio (SIR) values, than the stations in the closed vicinity of it. In this way, the throughput performance becomes poorer as the stations increase their distance from their AP.

Our goal is to propose the employment of a power control mechanism. We aim at mitigating the interference influence on network performance and at homogenizing the behavior of the different stations, independently of their distance from their AP.
For our study, we consider that all the stations in the system are working at the same data rate.

WLAN cards employ a link adaptation scheme that adapts their transmission rate to the $S I R$ experienced: when a station obtains low throughput performance, it decreases its transmission rate. Therefore, with the employment of a power control mechanism we pretend to improve the SIR performance of stations, so that a change in the transmission rate is not necessary, and, thus, stations will be able to work at higher transmission rates, and we will avoid the performance anomaly that appears in WLANs with hosts operating at different rates: the rate of a slower host limits the throughput of a fast host [4].

There are different ways to implement power control: mechanisms consisting on regulating manually the transmit power using the configuration software that most IEEE 802.11 hardware devices provide, and more complicated methods that would take into account the SIR measures and would define new IEEE 802.11 control messages. Our proposals do not modify or implement new control messages for the IEEE 802.11 standard. We aim at presenting power control mechanisms that can be easily included in the configuration software provided by the IEEE 802.11 hardware devices. Moreover, the power control proposals work in a fully distributed way and do not require the presence of a coordinator.

First, we propose a quite simple open-loop method, which works as follows: initially, all stations are transmitting data frames at the maximum power level of $30 \mathrm{dBm}$. In infrastructure networks, the APs transmit periodically control frames known as Beacons, employing the maximum power level. These frames announce the existence of a network and are an important part of many network maintenance tasks. Each station receives a Beacon frame with a different power level, depending on its distance from its AP. In this way, each station is able to obtain the loss value due to its relative position to the AP, where it is associated. Subsequently, each station knows its loss value and calculates the power level necessary at its output, so that its frames arrive at its AP with a fixed power level. We consider that a station is not allowed to transmit at a power level higher than $30 \mathrm{dBm}$. Considering that the sensitivity at the receiver takes $-65 \mathrm{dBm}$, we have chosen a fixed maximum power level value at the AP of $-53 \mathrm{dBm}$; the $12 \mathrm{~dB}$ margin is left to accommodate possible multipath loses and influence of the interference. The mobile station will transmit frames at the same power during all the time, until it changes its position. This method is quite easy to implement in real hardware.

Besides, we propose a more sophisticated closed-loop method, which follows the working procedure specified subsequently. This power control is based on the algorithms proposed for the thirdgeneration WCDMA system [9]. Our proposal works as follows. Initially, all stations are transmitting data frames at the maximum power level of $30 \mathrm{dBm}$. After each acknowledgment frame (ACK) successfully received, the station decreases its transmit power level by a value called step $\Delta$. Furthermore, every time that the retransmission timeout expires at the transmitter, its power level is increased by $\Delta$. Remember that a station is not allowed to transmit at a power level higher than $30 \mathrm{dBm}$. To achieve a faster convergence of the algorithm, we propose to employ an adaptive step. The step value adapts itself to the transmit power adjustment needed, avoiding, in this way, instability. In this case, the step is multiplied by $\alpha$, when $n_{1}$ consecutive successfully ACKs are 
received, or when the retransmission timeout expires $n_{l}$ consecutive times. Finally, the step is divided by $\beta$ if the transmitter alternates between an ACK successfully reception and a retransmission timeout expiration. To perform our study, we consider $\Delta=1 \mathrm{~dB}, \alpha=1.5, \beta=1.5$ and $n_{1}=2$. With these parameters choice we achieve a faster convergence. We have evaluated this power control mechanism working with other parameter values, and we have observed that the results are not better but the instability becomes higher.

Finally, we propose a second closed-loop method, which is also based on the algorithms exposed for the third-generation WCDMA system [9]. Our proposal works as follows. Initially, all stations are transmitting data frames at the maximum power level of $30 \mathrm{dBm}$. After each data frame successfully received, the receiver estimates the $S I R$ and checks if this value is higher or lower than a fixed threshold. A new field is included in the ACK frame; in order to implement the Transmit Power Control (TPC) algorithm and to notify the transmitter a request on transmit power increase or decrease. The new TPC field introduced in the ACK frame is 1 byte long. If the instantaneous $S I R$ value at the data frame is higher than the threshold, the TPC bits are set to " 0 ", requesting a transmit power decrease. On the other hand, if the SIR value is lower than the threshold, the TPC bits are set to "1", requesting a transmit power increase. In this way, the power level at the transmitter will be increased or decreased a value called step $\Delta$. Remember that a station is not allowed to transmit at a power level higher than $30 \mathrm{dBm}$. To achieve a faster convergence of the algorithm, we propose the employment of an adaptive step, as explained in previous paragraph. In this case, the step is multiplied by $\alpha$, when $n_{l}$ consecutive successfully ACKs with the TPC bits set to " 0 " are received, or when $n_{1}$ consecutive successfully ACKs with the TPC bits set to "1" are obtained. Finally, the step is divided by $\beta$, if the transmitter alternates between an ACK successfully reception with the TPC bits set to " 0 " and another with these bits set to " 1 ". To perform our study, we consider a $S I R$ threshold of $25 \mathrm{~dB}$ and $\Delta=1 \mathrm{~dB}, \alpha=1.5, \beta=1.5$ and $n_{l}=2$. With these parameters choice we achieve a faster convergence and lower instability, as explained in previous paragraph. Note that we employ a TPC field of 1 byte. In this way, in future implementations, it is possible to set different step values in relation to the difference between the current $S I R$ value and the threshold, and include the correspondent step value in the $T P C$ field of the ACK frame.

When working under any of the power control mechanisms exposed, the hidden terminal problem appears. This fact degrades IEEE 802.11 performance. In this way, we decide to employ the RTS/CTS working mechanism for the different power control methods proposed. Thus, if RTS and CTS frames are always transmitted at maximum power level, the hidden terminal problem is solved. Finally, note that a station transmits the data and ACK frames employing the same power level.

\section{SYSTEM PERFORMANCE}

Next, we evaluate the different power control mechanisms exposed in previous section. We present system performance under different load conditions per cell and compare the results obtained with the behavior observed in a cellular system working without any power control mechanism. Without a loss of generality, we employ a transmission data rate of $48 \mathrm{Mbps}$. The offered load per cell is presented as the normalized offered load for that transmission rate, in this case $48 \mathrm{Mbps}$. Moreover, note that the normalized saturation throughput for a single cell system operating at $48 \mathrm{Mbps}$ is 0.48 , and for $54 \mathrm{Mbps}$ it takes a value of 0.45 [8]. In this way, for our study, we consider normalized offered loads (OL) per cell of 0.3 and 0.4 .

We use a Fixed Channel Allocation (FCA) scheme with an initial cluster size of three cells and assign channels 1,6 and 11 of the $2.4 \mathrm{GHz}$ band, which do not overlap between them.

Figure 1 presents the average SIR value at AP for frames (RTS and data) generated by stations placed at different spatial positions with respect to their access point. Remember that the RTS frames are always sent at maximum power level. RTS frames sent by stations far away from the AP arrive at the access point with lower power level, thus, the average SIR value decreases with the rise of the distance from the AP. On the other hand, stations adapt their transmit power for the transmission of data frames. Employing either of the three power control mechanisms explained in previous section, the most distant stations improve their average $S I R$ in relation to the original network situation, as a consequence of the interference level reduction. On the other hand, the closer stations decrease their average $S I R$; due to the important reduction in the data frames transmit power.

Under the transmission conditions chosen and employing any of the power control methods proposed, the access points continue operating at maximum power. Access points are not sending data frames, and, in this way, no power control algorithm is applied at APs transmissions. Consequently, the ACK frames generated at each AP are received at stations with different power level, depending on the distance between each station and its AP, and, in this way, the average $S I R$ at the stations decreases with the rise of the distance (see Figure 2). Remember that the CTS frames are always transmitted at maximum power, as explained in previous section. The average SIR becomes higher in relation to the results obtained when the system operates without power control, due to the reduction of the overall interfering power level. Note that Figures 1 and 2 present $S I R$ performance for an offered load per cell of 0.3 . Results for $\mathrm{OL}=0.4$ are equivalent; we do not present them in this paper because of length restrictions.

From Figures 3 and 4 we can observe throughput performance for the stations placed at different spatial positions with respect to their AP, for various load conditions per cell and the different power control mechanisms proposed. When the system operates without power control, the stations at positions more far away from the AP decrease their throughput performance for increasing network load [8]. With the use of power control mechanisms, the interference level decreases and the average SIR at AP and at stations increases for the most distant stations. Transmit power can be decreased up to $0 \mathrm{dBm}$, which is the minimum transmission power fixed by the standard. In this way, the nearest station, which is placed at $15 \mathrm{~m}$ from the AP, decreases its transmission power to the minimum value, and it still maintains its throughput as high as in the original case. Unfortunately, the decrease in the interference level is insufficient to obtain a considerable improvement in the throughput performance of the most distant stations, as can be observed in Figures 3 and 4.

Moreover, the employment of the open-loop power control mechanism proposed achieves worse throughput performance for 
the closer stations. When the network is working under this power control mechanism, every mobile station is transmitting with a power value that allows that its frames arrive at the AP with a fixed power level. In this way, this mechanism does not adapt to the stations needs, and results reveal that the power level fixed at the AP receiver is insufficient, so that the closest stations are not able to maintain their throughput as high as in the original case, obtaining an important performance decrease. On the other hand, in this case, the most distant stations increase their throughput; due to the important reduction in the data frames transmit power of the closest stations.

Furthermore, the employment of the second closed-loop power control mechanism exposed, which takes into account the SIR value at the receiver, achieves a better throughput performance. With the use of a SIR threshold of $25 \mathrm{~dB}$ the closer stations maintain their throughput as high as in the original case and, besides, the overall interference power level is reduced considerably. Moreover, using this mechanism, the variation of the different station performances is smaller than the obtained with the employment of the first closed-loop proposal, which changes the transmit power for each ACK successfully reception and each retransmission timeout expiration.

Finally, note that employing the second closed-power control proposal, the station placed at $130 \mathrm{~m}$ from the AP achieves an important improvement in its throughput performance, when the offered load per cell is 0.4 (see Figure 4).

Subsequently, we evaluate the power control mechanisms proposed employing a cluster size of four cells. In this case, four partially overlapped frequencies are being used (channels 1, 4, 8 and 11), with adjacent-channel interference factors presented in [2]; at $2.4 \mathrm{GHz}$ only three non-overlapped channels can be employed.

We evaluate the power control mechanisms for this new scenario. From Figures 5 and 6 we can observe the throughput performance per station for different load conditions per cell. Employing a cluster size of four cells together with the power control mechanisms, we achieve a higher interference power level reduction, and, in this way, the throughput performance obtained is better than the observed for a cluster size of three cells. Besides, employing the open-loop mechanism, we observe an important throughput improvement. Using a cluster size of four cells, the power level fixed at the AP is more suitable, so that the closest stations improve their throughput performance.

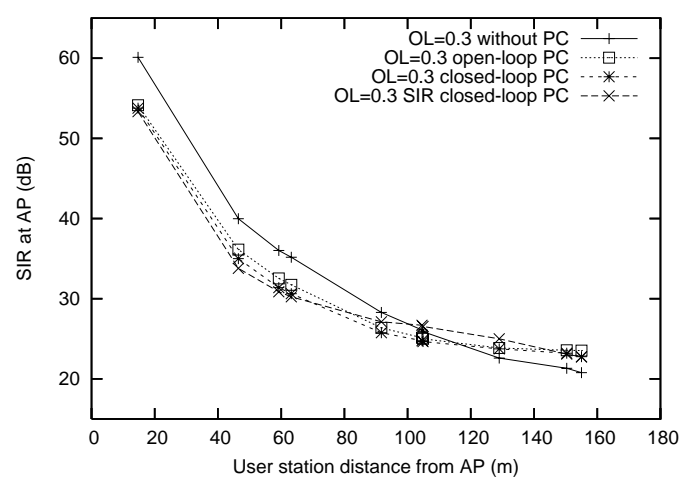

Figure 1. SIR at AP vs. station distance from its AP, for a cluster of 3 cells and offered load per cell of 0.3

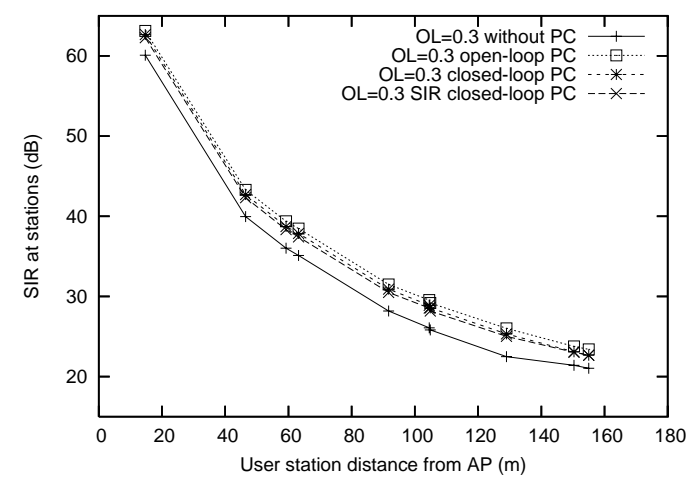

Figure 2. SIR at stations vs. station distance from its AP, for a cluster of 3 cells and offered load per cell of 0.3

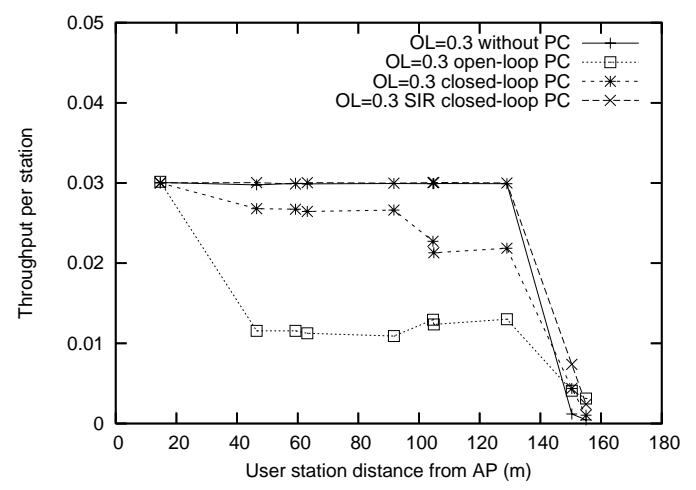

Figure 3. Throughput per station vs. station distance from its AP, for a cluster of 3 cells and offered load per cell of 0.3

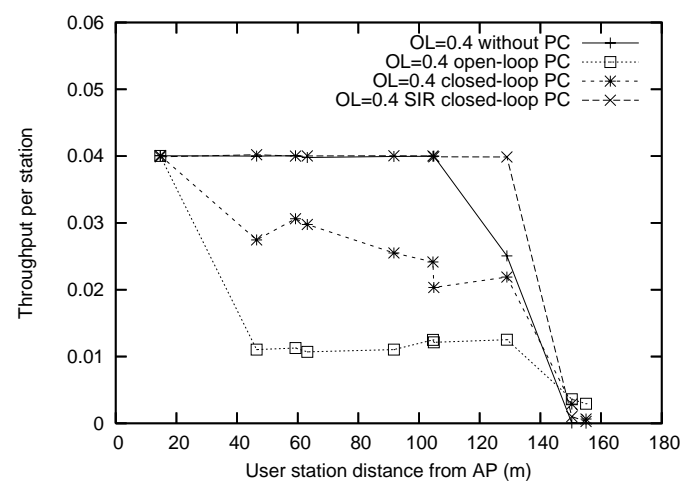

Figure 4. Throughput per station vs. station distance from its AP, for a cluster of 3 cells and offered load per cell of 0.4

Moreover, employing any of the closed-loop power control proposals, we achieve a better overall throughput performance, and the most distant stations improve their behavior. Using the second closed-loop proposal, the closer stations maintain their throughput as high as in the original case and, besides, the overall interference power level is reduced considerably. On the other hand, employing this mechanism, the most distant stations improve considerably their performance, in comparison with the original case and with the other two power control proposals exposed, when the mechanism is working under lower traffic load conditions (normalized offered load per cell of 0.3) (see Figure 5). When the system is working under higher traffic load conditions (see Figure 6), the most distant stations obtain a better 
performance employing the first closed-loop proposal, which changes the transmit power for each ACK successfully reception and each retransmission timeout expiration. Note that employing this mechanism, the closer stations reduce their throughput in comparison with the original case, due to an important decrease in their data transmit power. In this way, the most distant stations achieve an important throughput improvement, due to the interference level reduction obtained.

Finally, we evaluate both closed-loop proposals when stations are allowed to transmit data frames at a power higher than $30 \mathrm{dBm}$. Initially, all stations are sending data frames at a power level of $30 \mathrm{dBm}$; but they are allowed to increase this power up to $33 \mathrm{dBm}$. On the other hand control frames (RTS, CTS and ACK frames) are always sent at a maximum power of $30 \mathrm{dBm}$.

In Figures 7 and 8 we present the throughput performance per station for different load conditions per cell, and cluster size of four cells, when the system is employing the first closed-loop proposal, which changes the transmit power for each ACK successfully reception and each retransmission timeout expiration. We compare the results obtained when stations are allowed to transmit data frames up to a maximum power of $30 \mathrm{dBm}$ and when they are able to employ up to $33 \mathrm{dBm}$. The same evaluation is presented for the second closed-loop power control mechanism exposed, which takes into account the $S I R$ value at the receiver (see Figures 9 and 10).

Results show that the increase in the maximum transmission power of data frames achieves an important improvement in the throughput performance of the most distant stations (see Figures 7-10). Moreover, the closest stations only decrease slightly their performance, in comparison with the case employing $30 \mathrm{dBm}$ as maximum transmission power. In this way, adding this modification to the closed-loop power control algorithms, we achieve a better overall system performance.

As stated previously, control frames are always sent at a maximum power of $30 \mathrm{dBm}$. On the other hand, only the most distant stations need to increase their transmit power up to $33 \mathrm{dBm}$. As we have explained in previous paragraphs, allowing stations to rise transmit power for data frames up to $33 \mathrm{dBm}$, we obtain still a considerable interference level reduction. However, if we increase this maximum power level to higher values, we observe a rise in the interference level, what accentuates the overlapping cells problem and leads to a decrease in system performance.

\section{CONCLUSIONS}

In this paper, we present three power control mechanisms proposals. Our goal is to mitigate the interference influence on network performance and to homogenize the behavior of the stations placed at different spatial positions with respect to their access point. On the other hand, the employment of power control methods leads to an important reduction in the interference level what allows stations to work at higher data rates, and, thus, avoids the performance anomaly problem that appears in WLANs with hosts operating at different rates.

Results reveal that the use of the closed-loop power control mechanisms exposed achieves better system performance than the open-loop proposal. These mechanisms work in a fully distributed way, do not need the presence of a coordinator and adapt to the instantaneous needs of stations. Finally, increasing maximum transmit power up to $33 \mathrm{dBm}$, system exhibits a better overall performance.

\section{ACKNOWLEDGMENTS}

This research has been funded by FEDER and the Spanish Government through CICYT project TIC2003-01748.

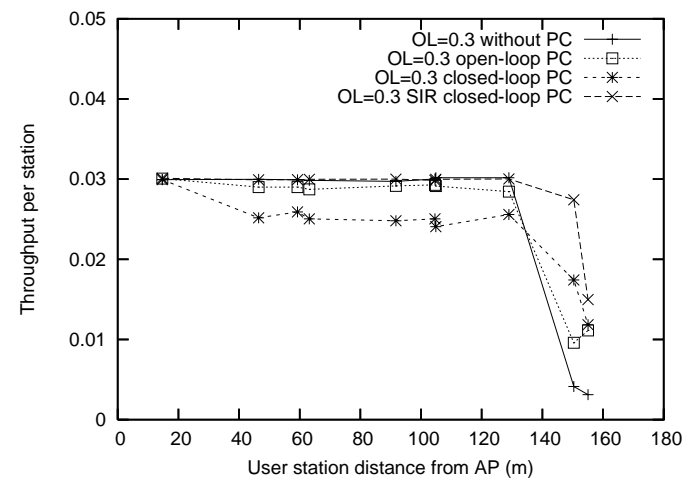

Figure 5. Throughput per station vs. station distance from its AP, for a cluster of 4 cells and offered load per cell of 0.3

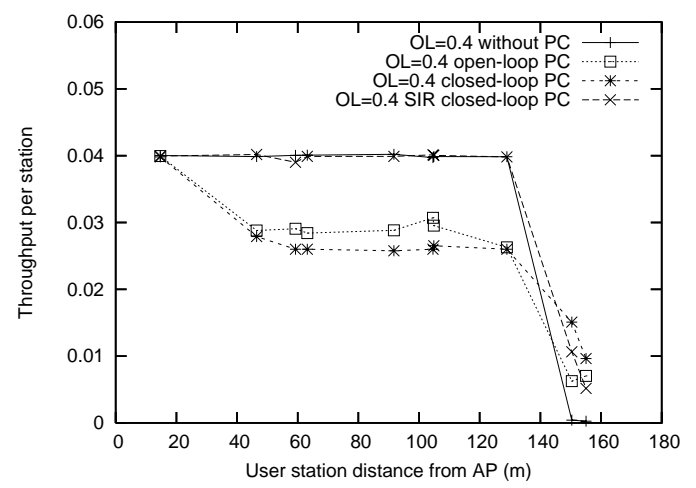

Figure 6. Throughput per station vs. station distance from its AP, for a cluster of 4 cells and offered load per cell of 0.4

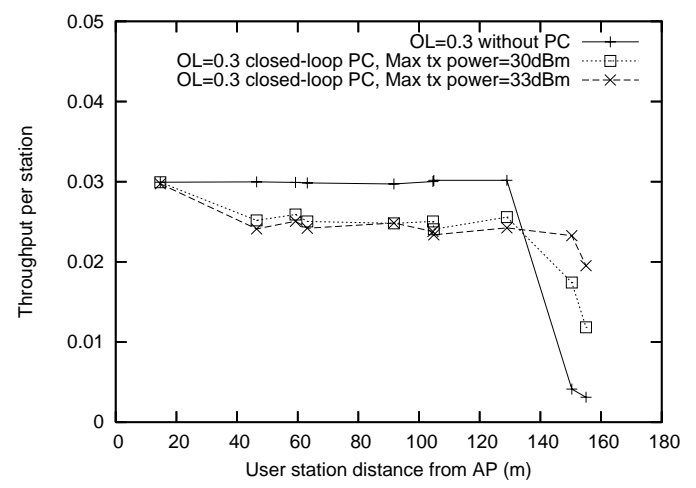

Figure 7. Throughput per station vs. station distance from its AP, for a cluster of 4 cells, offered load per cell of $0.3,1^{\text {st }}$ closed-loop power control mechanism 


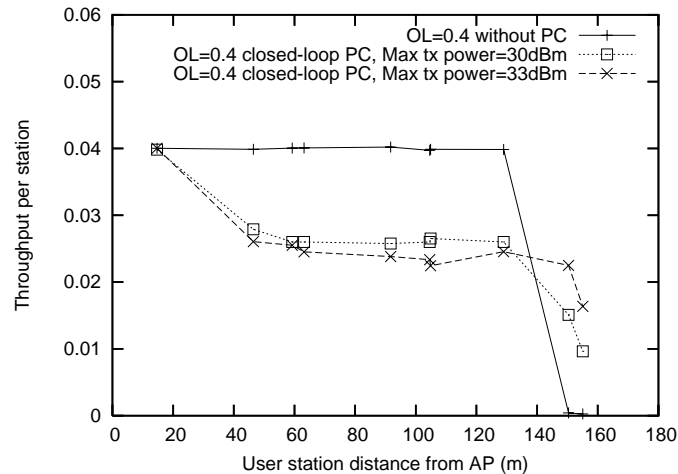

Figure 8. Throughput per station vs. station distance from its AP, for a cluster of 4 cells, offered load per cell of $0.4,1^{\text {st }}$ closed-loop power control mechanism

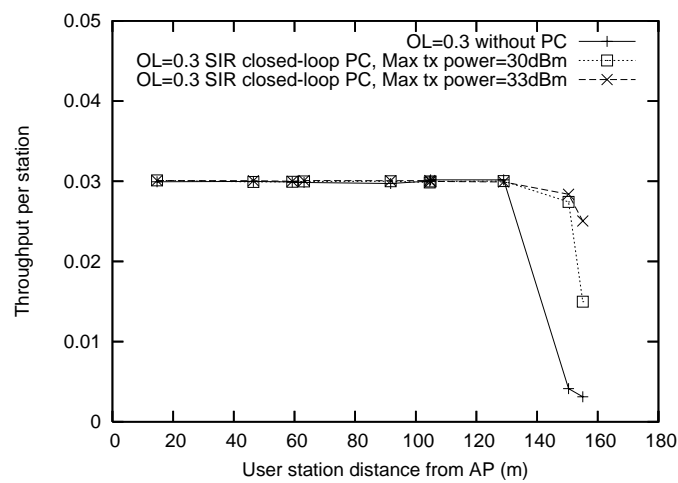

Figure 9. Throughput per station vs. station distance from its AP, for a cluster of 4 cells, offered load per cell of $0.3,2^{\text {nd }}$ closed-loop power control mechanism

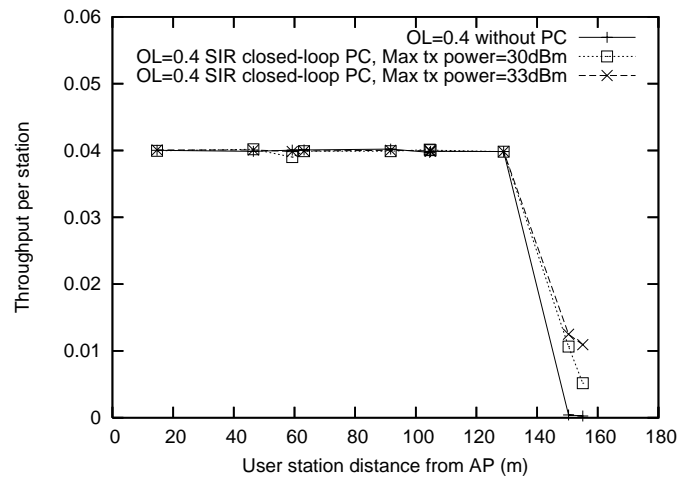

Figure 10. Throughput per station vs. station distance from its $A P$, for a cluster of 4 cells, offered load per cell of $0.4,2^{\text {nd }}$ closed-loop power control mechanism

\section{REFERENCES}

[1] Bianchi, G. Performance analysis of the IEEE 802.11 Distributed Coordination Function, IEEE Journal on selected areas in communications, Vol. 18, No. 3, pp. 535 - 547, March 2000.

[2] Burton, M. Channel Overlap Calculations for 802.11b Networks, White Paper, Cirond Networks Inc., February 2002.

[3] Green, D. B. and Obaidat, A. S. An accurate line of sight propagation performance model for ad-hoc 802.11 wireless LAN (WLAN) devices, Proc. of the IEEE International Conference on Communications 2002 (ICC 2002), Vol. 5, pp. 3424 - 3428, April 2002.

[4] Heusse, M., Rousseau, F., Berger-Sabbatel, G. and Duda, A. Performance Anomaly of $802.11 \mathrm{~b}$, Proc. of the IEEE INFOCOM 2003, Vol. 2, pp. 836-846, March-April 2003.

[5] Jelitto, J., Barreto, A. N. and Truong, H.L. Power and rate adaptation in IEEE 802.11a wireless LANs, Proc. of the IEEE Semiannual Vehicular Technology Conference 2003 (VTC 2003-Spring), Vol. 1, pp. 413 - 417, April 2003.

[6] Krunz, M. and Muqattash, A. A power control scheme for MANETs with improved throughput and energy consumption, Proc. of the International Symposium on Wireless Personal Multimedia Communications, Vol. 2, pp. 771 -775, October 2002.

[7] Leung, K. K., McNair, B., Cimini, L. J. and Winters, J. H. Outdoor IEEE 802.11 cellular networks: MAC protocol design and performance, Proc. of the IEEE International Conference on Communications 2002 (ICC 2002), Vol. 1, pp. 595 - 599, April 2002.

[8] Lopez-Aguilera, E., Casademont, J. and Cotrina, J. Outdoor IEEE $802.11 \mathrm{~g}$ cellular network performance, Proc. of the IEEE Global Telecommunications Conference (GLOBECOM 2004), Vol. 5, pp. 2992-2996, November - December 2004.

[9] Nuaymi, L., Lagrange, X. and Godlewski, P. A power control algorithm for 3G WCDMA system, Proc. of the European Wireless 2002, February 2002.

[10] Pursley, M. B. and Taipale, D. J. Error probabilities for spread-spectrum packet radio with convolutional codes and Viterbi decoding, IEEE Transactions on communications, Vol. COM-35, No. 1, pp. 1-12, Jan 1987.

[11] Qiao, D., Choi, S., Jain, A. and Shin, K. G. Adaptive transmit power control in IEEE 802.11a wireless LANs, Proc. of the IEEE Semiannual Vehicular Technology Conference 2003 (VTC 2003-Spring), Vol. 1, pp. 433-437, April 2003.

[12] Ruffini, M. and Reumerman, H.J. Power-rate adaptation in high-mobility distributed ad-hoc wireless networks, Proc. of the IEEE Semiannual Vehicular Technology Conference 2005 (VTC 2005-Spring), Vol. 4, pp. 2299 - 2303, May 2005.

[13] Wu, S.L., Tseng, Y.C. and Sheu, J.P. Intelligent medium access for mobile ad hoc networks with busy tones and power Control, IEEE Journal on Selected Areas in Communications, Vol. 18, No. 9, pp. 1647-1657, 2000. 